\title{
Intervención cognitivo-conductual para promover adherencia terapéutica y regulación autonómica en pacientes con diabetes mellitus tipo $2^{1}$
}

\author{
Cognitive-behavioral intervention \\ to promote therapeutic adherence and autonomic regulation \\ in patients with type-2 diabetes mellitus
}

\author{
Sandra Nayeli Vergara Aguirre ${ }^{2}$, Omar Arturo Chavarría Santiago ${ }^{3}$, \\ David Alberto Rodríguez Medina ${ }^{3}$, Benjamín Domínguez Trejo ${ }^{3}$ \\ y Patricia Zamudio Silva ${ }^{4}$
}

Citación: Vergara A., S.N., Chavarría S., O.A., Rodríguez M., D.A., Domínguez T., B. y Zamudio S., P. (2019). Intervención cognitivo-conductual para promover adherencia terapéutica y regulación autonómica en pacientes con diabetes mellitus tipo 2. Psicología y Salud, 29(2), 207-217.

\section{RESUMEN}

\begin{abstract}
La diabetes mellitus tipo 2 es una de las enfermedades no transmisibles que comprometen la actividad del sistema nervioso autonómico y que representa la principal causa de muerte en México. Actualmente, la terapia cognitivo-conductual provee la mayor evidencia empírica sobre su efectividad en el abordaje integral de la enfermedad. El propósito de esta investigación fue promover la adherencia terapéutica y regular la actividad autonómica en pacientes con dicha enfermedad a través de una intervención cognitivo-conductual grupal. Mediante un muestreo no probabilístico por conveniencia, participaron nueve mujeres, con una media de edad de 49.77 años y con diagnóstico confirmado de diabetes mellitus tipo 2, quienes recibieron dieciocho sesiones de terapia cognitivo-conductual. Se utilizó un diseño preexperimental de medidas psicofisiológicas y psicosociales repetidas. Las pacientes respondieron la Escala de Adherencia Terapéutica, el Cuestionario de Regulación Emocional, la Escala de Ansiedad y Depresión Hospitalaria y el Cuestionario de Apoyo Social. Para medir los cambios psicofisiológicos, se utilizó un equipo de retroalimentación biológica y un baumanómetro digital certificado. Los resultados sugieren que la terapia cognitivo-conductual permitió a las pacientes regular su actividad autonómica cardiovascular a través del aumento de la variabilidad de frecuencia cardíaca y la disminución de la presión arterial media, además de experimentar un aumento clínicamente significativo de su adherencia terapéutica. Asimismo, la terapia cognitivo-conductual fue efectiva para el manejo de los síntomas de ansiedad y depresión comórbidos y para el aumento de la percepción de las redes de apoyo. Por lo tanto, se concluye que la medición indirecta de la función autonómica mediante la variabilidad de frecuencia cardíaca y la presión arterial media es un parámetro objetivo útil para evaluar su influencia sobre la salud mental y física en pacientes con diabetes mellitus tipo 2 .
\end{abstract}

Palabras clave: Diabetes mellitus tipo 2; Variabilidad de la frecuencia cardíaca; Ansiedad; Apoyo social; Depresión; Regulación emocional.

\footnotetext{
${ }^{1}$ Los autores agradecen al Dr. Erick Leonardo Mateos Salgado y al Grupo de Investigación Clínica "Mente-Cuerpo" sus valiosos consejos. Con financiamiento del Proyecto UNAM-DGAPA-PAPIT IN304515: "Biomarcadores (autonómicos e inmunológicos) como indicadores del componente emocional en el dolor crónico" y del Proyecto PAPIME-DGAPA-UNAM PE300716: "Fronteras de la psicología: citocinas y emociones". Artículo recibido el 29 de agosto y aceptado el 5 de noviembre de 2018.

${ }^{2}$ Facultad de Estudios Superiores Zaragoza, Universidad Nacional Autónoma de México, Av. Guelatao 66, Col. Ejército de Oriente, Del. Iztapalapa, 09230 Ciudad de México, tel. (52)55-27-18-80-16, correo electrónico: zandravergara@gmail.com.

${ }^{3}$ Facultad de Psicología, Universidad Nacional Autónoma de México, Av. Universidad 3004, Col. Copilco Universidad, Del. Coyoacán, 04510 Ciudad de México, correos electrónicos: o.chavarria.santiago@gmail.com,psic.d.rodriguez@comunidad.unam.mx y benjamin@unam.mx. ${ }^{4}$ Hospital Regional “Gral. Ignacio Zaragoza”, ISsSTE, Calz. Ignacio Zaragoza, Chinam Pac de Juárez, 09208 Ciudad de México, correo electrónico: zamudio298@live.com.mx.
} 


\begin{abstract}
Type-2 diabetes mellitus, a non-communicable disease is the main cause of death in Mexico and it compromises the activity of the autonomic nervous system. Currently, cognitive-behavioral therapy provides more empirical evidence of its effectiveness within a comprehensive approach to type 2 diabetes. The purpose of the present study was to examine the effects of a cognitive-behavioral group intervention aimed at promoting therapeutic adherence and regulating the autonomic activity in patients with type-2 diabetes. A non-probabilistic convenience sampling procedure yielded nine women with average age of 49.77 years, with a confirmed diagnosis of type-2 diabetes, which were included in the study. Participants received 18 sessions of cognitive-behavioral therapy along a pre-experimental design including repeated psychophysiological and psychosocial measures. Participants answered the Therapeutic Adherence Scale, the Emotional Regulation Questionnaire, the Hospital Anxiety and Depression Scale, and the Social Support Questionnaire. Biofeedback equipment and a calibrated digital sphygmomanometer were used to measure psychophysiological changes. Results suggest that the cognitive-behavioral intervention allowed patients to regulate cardiovascular autonomic activity by increasing heart rate variability and decreasing mean arterial pressure. In addition, results show clinically significant improvement in therapeutic adherence as well as effective management of comorbid anxiety/depression symptoms and increased perceived support network. Therefore, it is concluded that indirect measurement of autonomic function through heart rate variability and mean arterial pressure are useful and objective parameters to evaluate mental and physical health in type-2 diabetic patients.
\end{abstract}

Key words: Type-2 diabetes mellitus; Heart rate variability; Social support; Anxiety; Depression; Emotional regulation.

\section{INTRODUCCIÓN}

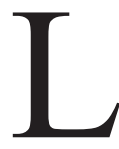

a Organización Mundial de la Salud (OMS) (2018) define como enfermedades no transmisibles (ENT) a las enfermedades que no se transmiten de persona a persona, que son de larga duración (o crónicas) y que resultan de una combinación de factores genéticos, fisiológicos, ambientales y conductuales. Una de las ENT con mayor crecimiento epidemiológico es la diabetes, definida como una "enfermedad crónica que aparece cuando el páncreas no produce insulina suficiente o cuando el organismo no utiliza eficazmente la insulina que produce" (OMS, 2017). De acuerdo con dicha organización, la diabetes se clasifica en diabetes tipo 1 (ausencia de síntesis de insulina), diabetes tipo 2 (incapacidad del cuerpo para utilizar eficazmente la insulina) y diabetes gestacional (hiperglicemia, que se detecta por primera vez durante el embarazo).

La octava edición del Atlas de Diabetes (International Diabetes Federation, 2017) estima que $8.8 \%$ de la población de entre 20 a 79 años sufre diabetes. De ésta, casi $80 \%$ se concentra en países en vías de desarrollo. Alrededor de $90 \%$ del total de personas con diabetes son diagnosticados con diabetes mellitus tipo 2 (DMT2 en lo sucesivo).

De acuerdo con la Encuesta Nacional de Salud y Nutrición hecha por el Instituto Nacional de Salud Pública (2016), 13 millones de mexicanos padecen DMT2, de los cuales solo aproximadamente la mitad han sido diagnosticados. Son las mujeres quienes padecen en mayor grado la diabetes, pero solamente $12.5 \%$ se encuentra bajo un control adecuado.

El tratamiento de la DMT2 implica modificar los comportamientos del paciente relacionados con su alimentación, actividad física, ingesta de medicamentos, monitoreo de su condición y demás, lo que implica una tarea compleja que propicia frecuentemente el abandono del tratamiento (Centro Nacional de Excelencia Tecnológica en Salud, 2014). Además, en muchos casos de DMT2 están presentes la depresión, la ansiedad y el estrés, lo que obstaculiza la adherencia al tratamiento. En contraste, el apoyo social percibido se asocia a menudo con un mejor control glucémico y calidad de vida, un mejor conocimiento de la enfermedad y una mayor adherencia al tratamiento. Para una mejor aceptación del diagnóstico son benéficos el ajuste emocional y la disminución del estrés (Strom y Egede, 2012).

Para el manejo de la DMT2 se han desarrollado diversas estrategias de control metabólico, aunque destacan las intervenciones psicoeducativas, las cuales generan resultados favorables en cuanto a la adquisición de conocimientos sobre la enfermedad, si bien no logran que los cambios experimentados se mantengan a través del tiempo (González y Oropeza, 2016). 
De acuerdo con diversos metaanálisis, el enfoque cognitivo-conductual ha mostrado tener una considerable eficacia (González y Oropeza, 2016; Li et al., 2017; Wang, Xia, Zhao y Chen, 2017) para que los pacientes con DMT2 desarrollen habilidades de afrontamiento, tales como aceptar la enfermedad, cumplir las demandas sistemáticas que impone la misma, descubrir las causas de la descompensación metabólica cuando se cumplen las indicaciones médicas, nutrirse adecuadamente, modular los temores e incertidumbres respecto a la salud presente y futura, y someterse al tratamiento de diferentes trastornos comórbidos cuyo impacto psicológico se refleja en el control metabólico.

Existen otras intervenciones terapéuticas que promueven la salud mediante la autorregulación, la meditación, el yoga, las técnicas de relajación y respiración, la hipnoterapia, la imaginería visual y la retroalimentación biológica, mismas que entrenan a las personas a reconocer ciertos estados mentales derivados de los procesos cerebrales y a tener una sensación de control sobre el cuerpo y el comportamiento. Tales intervenciones obtienen con frecuencia resultados confiables y válidos que demuestran su efectividad para aumentar la autoeficacia de los pacientes con ENT (Orozco y Castiblanco, 2015; Senders, Wahbeh, Spain y Shinto, 2012).

Sin embargo, el abordaje psicológico tradicional no ha incluido el uso de medidas psicofisiológicas para el entrenamiento de la regulación autonómica en estos pacientes, pese a que algunas medidas de dicha regulación, como la variabilidad de la frecuencia cardíaca (VFC en lo sucesivo), se utilizan en una amplia gama de enfermedades asociadas a una variabilidad disminuida, entre ellas la DMT2 y ciertos trastornos psiquiátricos, como la ansiedad y la depresión (Gernot, 2014a, 2014b).

Una revisión sistemática de la DMT2 hecha por Fakhrzadeh et al. (2012) concluyó que la VFC puede ayudar a predecir la morbilidad y la mortalidad cardíaca, y que puede usarse en una etapa temprana para indicar el riesgo futuro de complicaciones. Ya Yoshioka y Terasaki informaron en 1994 que el componente de alta frecuencia (HF, por sus siglas en inglés), asociada a la actividad nerviosa parasimpática en pacientes diabéticos, era más bajo que en sujetos de control y que había una correlación inversa entre la baja frecuencia (LF, por sus siglas en inglés) y la HF (cf. Young y Benton, 2018). Tales hallazgos sugieren que un deterioro del funcionamiento del sistema nervioso autónomo, reflejado en la VFC, ocurre durante las primeras etapas de la diabetes y empeora progresivamente en el tiempo.

También se ha encontrado una disfunción del sistema nervioso autónomo con una reducción asociada de la VFC en una amplia gama de trastornos psiquiátricos. Por ejemplo, Kemp et al. (2010) examinaron la asociación entre depresión y VFC en sujetos sin enfermedad cardiovascular. Cuando compararon a los pacientes con diagnóstico de depresión con un grupo control, hallaron que los primeros tenían una menor VFC; y que la misma era particularmente más baja en aquellos pacientes con síntomas más severos. Las revisiones metaanalíticas han mostrado reducciones de la VFC en los trastornos de ansiedad (Chalmers, Quintana, Abbott y Kemp, 2014), con un tamaño de efecto de pequeño a moderado.

En consecuencia, el propósito de este estudio prospectivo y preexperimental de medidas repetidas fue promover la adherencia terapéutica y la regulación de la actividad autonómica cardiovascular (decremento de la presión arterial media, disminución de la LF e incremento de la HF en la VFC) en pacientes con DMT2 a través de una intervención cognitivo-conductual grupal.

\section{MÉTODO}

\section{Participantes}

Seleccionadas de forma no probabilística y por conveniencia, se invitó a participar en el estudio a 16 pacientes ambulatorias con diagnóstico confirmado de DMT2, con una edad media de 49.77 años y desviación estándar de 11.32 años, y tiempo desde el diagnóstico de 11.4 años en promedio, las cuales asistían al Servicio de Endocrinología y Clínica de Diabetes del Hospital Regional "Gral. Ignacio Zaragoza" del Instituto de Seguridad Social al Servicio de los Trabajadores del Estado (ISSSTE). Todas ellas acudieron a la primera evaluación psicofisiológica y a la sesión grupal inicial; sin embargo, solamente nueve concluyeron la intervención 
psicológica debido a que las restantes abandonaron voluntariamente el estudio, por lo que no fueron consideradas en el análisis estadístico.

En la Tabla 1 se presenta la información sociodemográfica de la muestra resultante.

Tabla 1. Características sociodemográficas de la muestra.

\begin{tabular}{|c|c|c|c|}
\hline \multicolumn{2}{|c|}{ VARIABLES } & $\mathbf{N}$ & $\%$ \\
\hline \multirow{5}{*}{ Estado civil } & Separada & 2 & 22.2 \\
\hline & Divorciada & 1 & 11.1 \\
\hline & Casada & 2 & 22.2 \\
\hline & Soltera & 3 & 33.3 \\
\hline & Viuda & 1 & 11.1 \\
\hline \multirow{4}{*}{ Escolaridad } & Carrera técnica & 2 & 22.2 \\
\hline & Preparatoria & 1 & 11.1 \\
\hline & Licenciatura & 5 & 55.5 \\
\hline & Maestría & 1 & 11.1 \\
\hline \multirow{6}{*}{ Ocupación } & Jubilada & 2 & 22.2 \\
\hline & Auditora & 1 & 11.1 \\
\hline & Educadora & 1 & 11.1 \\
\hline & Ama de casa & 1 & 11.1 \\
\hline & Empleada federal & 2 & 22.2 \\
\hline & Enfermera & 2 & 22.2 \\
\hline \multirow{3}{*}{$\begin{array}{l}\text { Índice de masa } \\
\text { corporal }\end{array}$} & Obesidad & 4 & 44.4 \\
\hline & Sobrepeso & 4 & 44.4 \\
\hline & Normal & 1 & 11.1 \\
\hline \multirow{3}{*}{$\begin{array}{l}\text { Enfermedades } \\
\text { vasculares }\end{array}$} & Hipertensión arterial & 3 & 33.3 \\
\hline & Hipotensión arterial & 4 & 44.4 \\
\hline & Sin enfermedades vasculares & 2 & 22.2 \\
\hline \multirow{5}{*}{$\begin{array}{l}\text { Otros } \\
\text { diagnósticos }\end{array}$} & Dolor crónico & 2 & 22.2 \\
\hline & Pie diabético & 1 & 11.1 \\
\hline & Síndrome de Evans & 1 & 11.1 \\
\hline & Escoliosis & 1 & 11.1 \\
\hline & Sin otras comorbilidades & 4 & 44.4 \\
\hline
\end{tabular}

\section{Instrumentos}

El registro de las mediciones se llevó a cabo a través de los instrumentos que se describen a continuación:

Entrevista estructurada, hecha ex profeso para este estudio.

Escala de Adherencia Terapéutica (EAT). Diseñada por Soria, Vega y Nava (2009) para evaluar la adherencia terapéutica en pacientes con enferme- dades crónicas, sin estar dirigida a una enfermedad específica, permite detectar el cumplimiento de las recomendaciones médicas. Está basada en comportamientos explícitos contenidos en 21 ítems que se agrupan en tres factores: Control sobre la ingesta de medicamentos y alimentos, Seguimiento médico-conductual y Autoeficacia. Cada factor consta de siete reactivos; a su vez, cada uno de los ítems contiene una escala de 0 a 100 en la que el paciente elige la efectividad de su comportamiento en términos de porcentajes. El coeficiente alfa de Cronbach $(\alpha)$ de la escala es de .92, siendo la fiabilidad final para cada ítem de .88 .

Cuestionario de Regulación Emocional (ERQ). Elaborado por Gross y John (2003), se emplea para evaluar las diferencias individuales en el uso de dos estrategias de regulación emocional: reevaluación cognitiva y supresión expresiva. Está constituido por diez ítems tipo Likert distribuidos en dos escalas para medir el grado de acuerdo respecto al uso de ambas estrategias. Presenta una consistencia interna de 0.79 para la reevaluación cognitiva y de 0.73 para la supresión expresiva, y una confiabilidad test-retest de 0.69 (Cabello, Salguero, Fernández y Gross, 2013).

Escala de Ansiedad y Depresión Hospitalaria (HADS). Desarrollada por Zigmond y Snaith en 1983 (cf. López et al., 2002), puede utilizarse para evaluar la presencia y severidad de síntomas ansiosos y depresivos en pacientes que acuden regularmente a medios hospitalarios no psiquiátricos o de atención primaria. Considera ciertas dimensiones cognitivas y afectivas y omite aspectos somáticos tales como insomnio, fatiga, pérdida de apetito y otros, evitando así atribuirlos a la enfermedad. Este instrumento está constituido por 14 ítems, de los cuales los siete reactivos nones corresponden a una sintomatología ansiosa y los siete pares a una depresiva, con consistencias internas de 0.84 y 0.86 , respectivamente, y un coeficiente de correlación intraclase de 0.946 .

Cuestionario de Apoyo Social (MOS-SSS). Creado por Sherbourne y Stewart (1991) para evaluar las características cuantitativas de la red de apoyo social, tales como tamaño y densidad, así como los efectos o consecuencias que le brindan al sujeto 
el acceso y conservación de las relaciones sociales que tiene en su red, está constituido por veinte ítems distribuidos en dos áreas: apoyo social estructural (un ítem de respuesta abierta) y apoyo social funcional (19 ítems de respuesta múltiple). Muestra una confiabilidad de 0.97 , y en las dimensiones, de entre 0.91 y 0.96 .

\section{Materiales}

Equipo de retroalimentación biológica computarizado de cuatro canales ProComp 2 Infiniti. De la

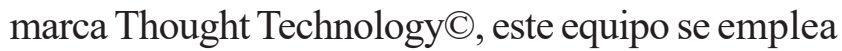
para decodificar y realizar el registro continuo de la VFC del paciente durante las evaluaciones psicofisiológicas.

Baumanómetro digital Citizen CH-656C. El dispositivo registra la presión arterial y el pulso. Para su uso, se consideraron los parámetros de la Norma Mexicana de Presión Arterial; en el caso presente, se utilizó la presión arterial media (PAM).

\section{Procedimiento}

La intervención psicológica se llevó a cabo durante un periodo de nueve meses y a lo largo de dieciocho sesiones.

En primera instancia, el protocolo de investigación fue evaluado y aprobado por el Comité de Ética y de Investigación del Hospital Regional "Gral. Ignacio Zaragoza" del ISSSTE.

Luego, el servicio de Endocrinología y Clínica de Diabetes identificó a las pacientes ambulatorias que potencialmente reunían los criterios para ser parte del grupo. Tras identificar a las pacientes y reunir los datos de cada una, la información obtenida fue enviada al servicio de Psicología, que se encargó de ponerse en contacto con ellas para invitarlas a formar parte del grupo terapéutico $y$, en caso de que aceptaran, a asistir a la primera sesión grupal que se llevaría a cabo en las aulas del hospital.

En la sesión de bienvenida se les informó la naturaleza del estudio, indicando que la intervención psicoterapéutica estaría divida en dos modalidades: una de quince sesiones grupales y otra de tres sesiones de mediciones psicofisiológicas. Si las pacientes aceptaban participar, firmaban un formato de consentimiento informado (véase Apéndice) antes de presentarse a las sesiones en las instalaciones del hospital para recibir el tratamiento psicológico.

El protocolo de evaluación e intervención se estructuró de la siguiente manera:

1. Evaluación psicométrica y psicofisiológica: Se aplicaron los instrumentos EAT, ERQ, HADS y MOS en las sesiones 2, 9 y 18 . Como recomendaciones previas, las pacientes no debían tomar café $\mathrm{u}$ otros estimulantes 24 horas antes de cada medición. Todas las evaluaciones se realizaron entre las 8 y las 12 horas del día.

Tras dar la bienvenida a la paciente, se le indicaba que tomara asiento para que el psicólogo llevara a cabo el registro de su frecuencia cardíaca (FC), presión arterial sistólica (PAS) y presión arterial diastólica (PAD) mediante el baumanómetro colocado a la altura de la muñeca del brazo izquierdo; acto seguido, se anotaba el resultado en la hoja de recolección de datos. Luego, el terapeuta limpiaba el dedo índice de la mano izquierda de la paciente y colocaba el sensor de BVP para medir en tiempo real la VFC. Una vez conectado adecuadamente el sensor de la paciente al equipo de retroalimentación biológica, el terapeuta obtenía el perfil psicofisiológico de respuesta al estrés dando a la paciente la siguiente indicación: "Coloque ambos pies sobre el piso y siéntese cómodamente con la espalda recargada correctamente sobre el respaldo de la silla. Manténgase inmóvil y en silencio a menos de que se le solicite lo contario. En caso de sentir alguna molestia durante la evaluación, ésta puede terminar cuando usted lo desee".

El mencionado perfil psicofisiológico de respuesta al estrés estuvo conformado por las siguientes condiciones:

a) Sentada con los ojos abiertos (SOA): Se le pidió a la paciente que se mantuviera sentada y con los ojos abiertos durante tres minutos.

b) Sentada con los ojos cerrados (SOC): Se le pidió a la paciente que se mantuviera sentada con los ojos cerrados durante tres minutos.

c) Evocación del estresor con los ojos cerrados (Es): Se le pidió a la paciente que evocara alguna situación de estrés o ansiedad que hubiera experimentado en los últimos días. 
d) Respuesta natural de relajación (RNR): $\mathrm{Se}$ le pidió a la paciente que tratara de relajarse como normalmente lo hacía, pero manteniendo los ojos cerrados durante el procedimiento.

Cada fase duró tres minutos, esto es, un total de doce minutos en las cuatro condiciones.

Al término de la evaluación se indicó a la paciente que abriera lentamente los ojos hasta volver a situarse en el contexto del consultorio, hecho lo cual se le preguntaba cómo se sentía y se le retiraba el sensor de BVP. Se le tomaban nuevamente las medidas de FC, PAS y PAD colocando el baumanómetro a la altura de la muñeca del brazo izquierdo y anotándose el resultado en la hoja de recolección de datos. Finalmente, se resolvían las dudas de la paciente, se le agradecía su colaboración y un terapeuta la acompañaba a la salida.

2. Intervención grupal cognitivo-conductual. Tal intervención se llevó a cabo de forma grupal en consultas de 120 minutos cada dos o tres semanas y a lo largo de 18 sesiones en las aulas del hospital. Las técnicas del programa de intervención psicológica se muestran en la Tabla 2.

Tabla 2. Técnicas cognitivo-conductuales de intervención psicológica.

\begin{tabular}{|c|l|}
\hline $\begin{array}{c}\text { Número } \\
\text { de sesión }\end{array}$ & \multicolumn{1}{c|}{ Técnica o estrategia cognitivo-conductual } \\
\hline 1 & Firma de consentimiento informado y sesión de bienvenida. \\
\hline 2 & Primera evaluación psicofisiológica. \\
\hline 3 & Psicoeducación. \\
\hline 4 & Técnicas de relajación. \\
\hline 5 & Técnicas de relajación: respiración diafragmática. \\
\hline 6 & Regulación emocional. \\
\hline 7 & Inoculación del estrés. \\
\hline 8 & Identificación de redes de apoyo social. \\
\hline 9 & Segunda evaluación psicofisiológica. \\
\hline 10 & Entrenamiento en habilidades sociales. \\
\hline 11 & Entrenamiento en habilidades sociales: respuesta asertiva. \\
\hline 12 & Modelo ABC. \\
\hline 13 & Modelo ABC. \\
\hline 14 & Ideas irracionales. \\
\hline 15 & Ideas irracionales. \\
\hline 16 & Técnicas de habilidades de afrontamiento y solución de problemas. \\
\hline 17 & Técnicas de habilidades de afrontamiento y solución de problemas. \\
\hline 18 & Tercera evaluación psicofisiológica. \\
\hline
\end{tabular}

\section{Análisis estadísticos}

Se extrajeron los datos descriptivos de las medidas psicométricas y psicofisiológicas. Se exploró la normalidad de los datos con una prueba estadística Shapiro-Wilk $(p<.05)$. Debido a que no se encontró una distribución normal, se utilizaron pruebas estadísticas no paramétricas de Wilcoxon para evaluar las diferencias pre-peri, peri-post y pre-post de las mediciones psicométricas y psico- fisiológicas. Se consideró un nivel de significancia de $\leq .05$.

\section{RESULTADOS}

La Figura 2 ilustra los cambios psicofisiológicos en la presión arterial media (PAM) y la variabilidad de la frecuencia cardíaca (VFC). 
Figura 2. Resultados de las evaluaciones psicofisiológicas a lo largo de la intervención. Las gráficas muestran a) cambios en la presión arterial media (PAM) y b) cambios en la distribución de las bandas de frecuencia de la VFC: proporción VLF/LF/HF (\%).

$\mathbf{a}$

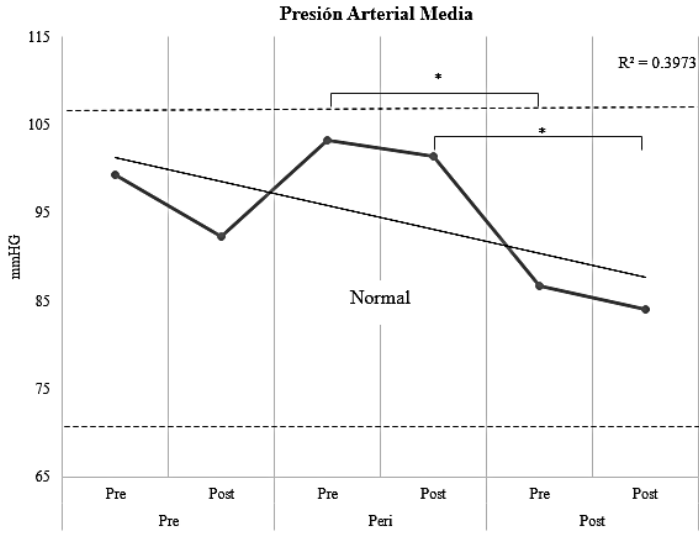

b

Distribución de las bandas de frecuencia de la VFC y proporción

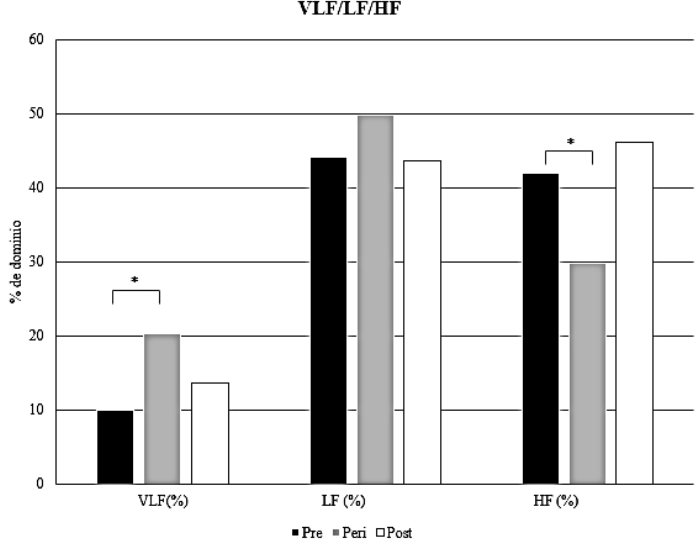

$* p<0.05$.

Los cambios estadísticamente significativos registrados en la PAM fueron los que se muestran en la Figura 2a: un decremento de la preevaluación de la sesión 9 a la preevaluación de la sesión 18 $(\mathrm{Z}=-2.201, p=.028)$, y de la postevaluación de la sesión 9 a la postevaluación de la sesión 18 $(\mathrm{Z}=-2.366, p=.018)$. La línea de tendencia central ilustra un decremento de la PAM a lo largo del tratamiento, con $\mathrm{R}^{2}=0.39$.

En la VFC (Figura 2b) se obtuvieron cambios estadísticamente significativos a lo largo de la intervención $\left(\mathrm{X}^{2}=114.525, p=.000\right)$. De acuerdo a lo anterior, se presentó dominancia de LF en la preevaluación (44.14\%) y en la perievaluación (49.80\%); en la postevaluación la dominancia correspondió a HF (46.15\%). No obstante, las bandas de porcentaje de frecuencia mostraron una disminución estadísticamente significativa en la HF de la preevaluación a la perievaluación $(\mathrm{Z}=-2.000$, $p=.046)$ y un aumento estadísticamente significativo de la VLF de la preevaluación a la perievaluación $(\mathrm{Z}=-2.057, p=.040)$.

La Figura 3 muestra las evaluaciones psicosociales obtenidas.

Es posible apreciar que $(a)$ la adherencia al tratamiento aumentó y resultó estadísticamente significativa de la preevaluación a la postevaluación $(\mathrm{Z}=-1.960, p=0.050)$. (b) La reevaluación cognitiva aumentó asimismo, aunque no hubo cambios estadísticamente significativos en ninguna de las mediciones: preevaluación-perievaluación $(\mathrm{Z}=-.210, p=0.833)$, perievaluación-postevaluación $(\mathrm{Z}=-.847, p=0.397)$ y preevaluación-postevaluación $(Z=-.282, p=0.778)$. (c) La supresión emocional mostró una tendencia a la baja, sin resultar estadísticamente significativa: preevalua- 
Figura 3. Evaluación psicosocial de la intervención.

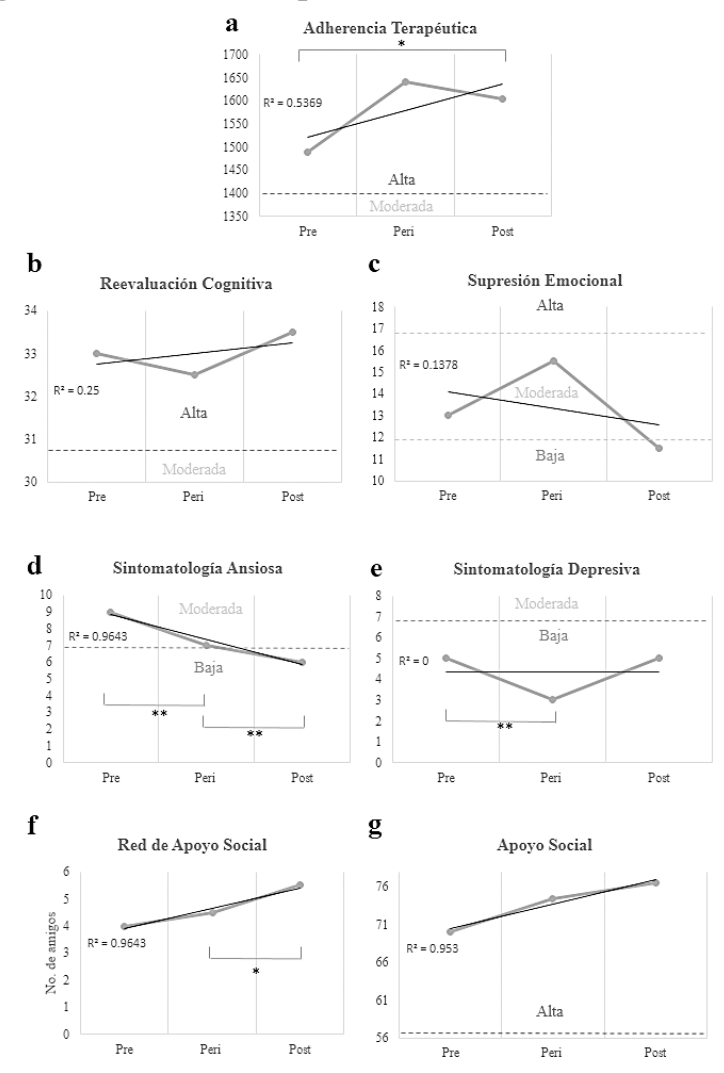

Las líneas punteadas representan los puntos de corte de cada escala, y la línea sólida es la tendencia de cada medida con su respectiva $\mathrm{R}^{2}$. Las gráficas muestran la mediana de las variables Adherencia terapéutica $(a)$, Reevaluación cognitiva $(b)$, Supresión emocional $(c)$, Sintomatología ansiosa $(d)$, Sintomatología depresiva (e), Red de apoyo social $(f)$ y Apoyo social $(g)$.

$* p<0.05, * * p<0.01$.

ción-perievaluación $(\mathrm{Z}=-.213, p=0.832)$, perievaluación-postevaluación $(\mathrm{Z}=-.738, p=0.461)$ $\mathrm{y}$ preevaluación-postevaluación $(\mathrm{Z}=-.813$, $p=0.416)$. (d) La sintomatología ansiosa disminuyó de la preevaluación a la perievaluación con significancia estadística $(\mathrm{Z}=-2.521, p=0.012)$, y de la perievaluación a la postevaluación hubo una disminución con resultados estadísticamente significativos $(\mathrm{Z}=-2.366, p=0.018)$. (e) Del mismo modo, la sintomatología depresiva mostró cambios estadísticamente significativos, decrementándose de la preevaluación a la perievaluación $(\mathrm{Z}=-2.388, p=0.017)$. (f) La red de apoyo social mostró un aumento estadísticamente significativo de la perievaluación a la postevaluación $(\mathrm{Z}=-2.041, p=0.041)$. ( $g$ ) Por último, el apoyo social percibido aumentó, pero esos incrementos no fueron estadísticamente significativos: preevaluación-perievaluación ( $\mathrm{Z}=-.281, p=0.779)$, perievaluación-postevaluación $(\mathrm{Z}=-1.363$, $p=0.173)$ y preevaluación-postevaluación $(\mathrm{Z}=-.561, p=0.574)$.

\section{DISCUSIÓN}

La DMT2 se asocia a una disminución de la actividad parasimpática y a una activación de la simpática debido a que es una enfermedad metabólica que conduce a una desregulación autonómica (Benichou et al., 2018).

Es importante resaltar que durante la intervención se presentó una variable extraña, la cual influyó en el tono simpático: el sismo del 19 de septiembre de 2017, una catástrofe natural, que por sí misma indujo un estado de alerta en las personas que la experimentaron. Un suceso así promueve un aumento de la actividad simpática y una disminución de la actividad parasimpática, lo que sugiere una tendencia a la baja de la regulación autonómi- 
ca observada hacia la novena sesión de la intervención; sin embargo, los resultados del estudio mostraron que los pacientes con DMT2 lograron recuperar la caída de VFC hacia el final del tratamiento debido al entrenamiento en regulación autonómica.

Una elevada VFC se asocia con mayor bienestar físico y mejor regulación emocional (Mather y Thayer, 2018). La inducción de oscilaciones de gran amplitud en la frecuencia cardíaca suprime la acción simpática al tiempo que estimula la acción parasimpática (Goessl, Curtiss y Hofmann, 2017), lo que explica la reducción de los síntomas de ansiedad, depresión y estrés; del mismo modo, la calidad de las interacciones sociales influye para predecir el control autonómico cardíaco (Gerteis y Schwerdtfeger, 2016). Es por ello que los resultados de la presente investigación subrayan la necesidad de considerar la interacción de los factores psicológicos y sociales para evaluar el efecto benéfico de las intervenciones psicológicas en la regulación autonómica cardiovascular.

En los países desarrollados, la tasa de adherencia a los tratamientos en el caso de enfermedades crónicas se sitúa alrededor de $50 \%$. Esta cifra es considerablemente menor en los países en vías de desarrollo, según un informe de la Organización Mundial de la Salud (OMS, 2016), que califica la falta de adherencia como un problema mundial de gran magnitud. Por ello, las intervenciones con enfoque cognitivo-conductual pretenden dotar a las personas de las habilidades necesarias para lograr dicha adherencia (González y Oropeza, 2016). No obstante, es necesario que esas intervenciones se enfoquen en estrategias que promuevan la regulación autonómica en estos pacientes en virtud de la creciente comorbilidad entre los problemas cardiovasculares y la DMT2, comorbilidad que podría provocar una muerte prematura (Arrieta et al., 2015).
Con base en los resultados de la evaluación psicosocial, se destaca que aunque no hubo diferencias estadísticamente significativas en la Escala de Regulación Emocional, se muestra sin embargo una tendencia clínicamente favorable para las pacientes con un nivel alto de reevaluación cognitiva y supresión emocional disminuida, lo que concuerda con su impacto en el aumento de la red de apoyo social. Asimismo, el puntaje de ansiedad disminuyó en una proporción estadísticamente significativa en este grupo. Finalmente, a pesar de que el puntaje de depresión se redujo de la sesión 1 a la 9 y luego volvió a aumentar, se mantuvo en un nivel bajo, y por ende no sería objeto de atención clínica.

Por último, respecto a las limitaciones del presente trabajo, debe decirse que no se contó con un tamaño de muestra aleatorizado y representativo de la población, ni tampoco con un grupo control, por lo que los resultados aquí mostrados únicamente responden al comportamiento de la muestra empleada. Se sugiere incorporar un periodo de seguimiento, así como otros indicadores clínicos, como HbAlc, colesterol y triglicéridos, para evaluar el control metabólico de los pacientes a lo largo de la intervención psicológica.

Se concluye que la intervención psicológica permitió a las participantes regular su actividad autonómica cardiovascular y aumentar clínicamente su adherencia terapéutica. La terapia cognitivo-conductual en pacientes con DMT2 fue efectiva para el manejo de los síntomas de ansiedad y depresión comórbidos y para el aumento de la percepción de las redes de apoyo. Así, es posible concluir que la medición indirecta de la función autonómica mediante la variabilidad de la frecuencia cardíaca y la presión arterial media es un parámetro objetivo útil para evaluar su influencia en la salud mental y física.

\section{REFERENCIAS}

Arrieta, F., Iglesias, P., Pedro-Botet, J., Tébar, F., Ortega, E. y Nubiola, A. (2016). Diabetes mellitus y riesgo cardiovascular: Recomendaciones del Grupo de Trabajo Diabetes y Enfermedad Cardiovascular de la Sociedad Española de Diabetes. Clínica e Investigación en Arteriosclerosis, 27(4), 181-192. doi: 10.1016 / j.arteri.2014.12.003.

Benichou, T., Pereira, B., Mermillod, M., Tauveron, I., Pfabigan, D., Maqdasy, S. y Dutheil, F. (2018). Heart rate variability in type 2 diabetes mellitus: A systematic review and meta-analysis. PLOS One, 13(4), 1-19. doi: 10.1371/journal.pone.0195166.

Cabello, R., Salguero, J., Fernández B., P. y Gross, J. (2013). A Spanish adaptation of the Emotion Regulation Questionnaire. European Journal of Psychological Assessment, 29(4), 234-240. doi: 10.1027/1015-5759/a000150. 
Centro Nacional de Excelencia Tecnológica en Salud (2014). Tratamiento de la diabetes mellitus tipo 2 en el primer nivel de atención. México: Instituto Mexicano del Seguro Social.

Chalmers, J., Quintana, D., Abbott, M. y Kemp, A. (2014). Anxiety disorders are associated with reduced heart rate variability: a meta-analysis. Frontiers in Psychiatry, 5, 1-11. doi: 10.3389/fpsyt.2014.00080.

Fakhrzadeh, H., Yamini-Sharif, A., Sharifi, F., Tajalizadekhoob, Y., Mirarefin, M. y Mohammadzadeh, M. (2012). Cardiac autonomic neuropathy measured by heart rate variability and markers of subclinical atherosclerosis in early type 2 diabetes. ISRN Endocrinology, 1-7. doi: 10.5402/2012/168264.

Gernot, E. (2014). Diabetes. En E. Gernot (Ed.): Heart rate variability (pp. 289-297). London: Springer. doi: 10.1007/9781-4471-4309-3_15.

Gernot, E. (2014). Other studies. En E. Gernot (Ed.): Heart Rate Variability (pp. 299-309). London: Springer. doi: 10.1007/9781-4471-4309-3_15.

Gerteis, A. y Schwerdtfeger, A. (2016). When rumination counts: Perceived social support and heart rate variability in daily life. Psychophysiology, 53(7), 1034-1043. doi: 10.1111 / psyp.12652.

Goessl, V., Curtiss, J. y Hofmann, S. (2017). The effect of heart rate variability biofeedback training on stress and anxiety: a meta-analysis. Psychological Medicine, 47(15), 2578-2586. doi: 10.1017/s0033291717001003.

González, J. y Oropeza, R. (2016). Intervenciones cognitivo conductuales para diabéticos en México. Salud Mental, 39(2), 99-105. doi: 10.17711/SM.0185-3325.2016.006.

Gross, J.J. y John, O.P. (2003). Individual differences in two emotion regulation processes: Implications for affect, relationships, and well-being. Journal of Personality and Social Psychology, 85, 348-362.

Instituto Nacional de Salud Pública (2016). Encuesta Nacional de Salud y Nutrición de Medio Camino 2016. Informe final de resultados, 2016. Recuperado de: http://ensanut.insp.mx/ensanut2016/index.php.

Internacional Diabetes Federation (2017). Diabetes Atlas 2017. Watermael-Boitsfort (Bélgica): IDF. Recuperado de http://fmdiabetes.org/wp-content/uploads/2018/03/IDF-2017.pdf.

Kemp, A., Quintana, D., Gray, M., Felmingham, K., Brown, K. y Gatt, J. (2010). Impact of depression and antidepressant treatment on heart rate variability: a review and meta-analysis. Biological Psychiatry, 67(11), 1067-1074. doi: 10.1016/j. biopsych.2009.12.012.

Li, C., Xu, D., Hu, M., Tan, Y., Zhang, P., Li, G. y Chen, L. (2017). A systematic review and meta-analysis of randomized controlled trials of cognitive behavior therapy for patients with diabetes and depression. Journal of Psychosomatic Research, 95, 44-54. doi: 10.1016/j.jpsychores.2017.02.006.

López, J., Vázquez, V., Arcila, D., Sierra, A., González, J. y Salín, R. (2002). Exactitud y utilidad diagnóstica del Hospital Anxiety and Depression Scale (HAD) en una muestra de sujetos obesos mexicanos. Revista de Investigación Clínica, 54(5), 403-409.

Mather, M. y Thayer, J. (2018). How heart rate variability affects emotion regulation brain networks. Current Opinion in Behavioral Sciences, 19, 98-104. doi: 10.1016/j.cobeha.2017.12.017.

Organización Mundial de la Salud (2016). Informe mundial sobre la diabetes. Ginebra: OMS. Recuperado de:http://apps.who. int/iris/bitstream/handle/10665/204877/WHO_NMH_NVI_16.3_spa.pdf;jsessionid = F3494143D48F29C1DE697289F972C88B?sequence $=1$.

Organización Mundial de la Salud, (2017). Diabetes. Ginebra: OMS. Recuperado de: http://www.who.int/es/news-room/factsheets/detail/diabetes.

Organización Mundial de la Salud (2018). Enfermedades no transmisibles. Ginebra: OMS. Recuperado de: http://www.who.int/es/ news-room/fact-sheets/detail/noncommunicable-diseases.

Orozco G., A. y Castiblanco O., L. (2015). Factores psicosociales e intervención psicológica en enfermedades crónicas no transmisibles. Revista Colombiana de Psicología, 24(1), 203-217. doi: 10.15446 / rcp.v24n1.42949.

Senders, A., Wahbeh, H., Spain, R. y Shinto, L. (2012). Mind-body medicine for multiple sclerosis: a systematic review. Autoimmune Diseases, 567324. doi: 10.1155/2012/567324.

Sherbourne, C. y Stewart, A. (1991). The Mos Social Support Survey. Social Science \& Medicine, 32(6), 705-714. doi: 10.1016/0277-9536(91)90150-b.

Soria, R., Vega, Z. y Nava, C. (2009). Escala de adherencia terapéutica para pacientes con enfermedades crónicas, basada en comportamientos explícitos. Alternativas en Psicología, 14(20), 89-103.

Strom, J. y Egede, L. (2012). The impact of social support on outcomes in adult patients with type 2 diabetes: a systematic review. Current Diabetes Reports, 12(6), 769-781. doi: 10.1007/s11892-012-0317-0.

Wang, Z., Xia, Y., Zhao, Y. y Chen, L. (2017). Cognitive behavioural therapy on improving the depression symptoms in patients with diabetes: a meta-analysis of randomized control trials. Bioscience Reports, 37(2), 1-6. doi: 10.1042/bsr20160557.

Yoshioka, K. y Terasaki, J. (1994). Relationship between diabetic autonomic neuropathy and peripheral neuropathy as assessed by power spectral analysis of heart rate variations and vibratory perception thresholds. Diabetes Research and Clinical Practice, 24(1), 9-14.

Young, H. y Benton, D. (2018). Heart-rate variability. A biomarker to study the influence of nutrition on physiological and psychological health? Behavioural Pharmacology, 29, 140-151. doi: 10.1097/fbp.0000000000000383.

Zigmond, A.S. y Snaith, R.P (1983). The Hospital Anxiety and Depression Scale. Acta Psychiatrica Scandinavica, 67, 361-370. 
Apéndice

Consentimiento informado

Intervención grupal cognitivo-conductual para la promoción de adherencia terapéutica y regulación autonómica en pacientes con diabetes mellitus tipo 2

Financiadores del proyecto:

Proyecto UNAM-DGAPA-PAPIIT IN304515: "Biomarcadores (autonómicos e inmunológicos) como indicadores del componente emocional en el dolor crónico".

Proyecto PAPIME-DGAPA-UNAM PE300716: "Fronteras de la psicología: citocinas y emociones".

Institución: Universidad Nacional Autónoma de México

Lugar: Hospital Regional “Gral. Ignacio Zaragoza”, ISSSTE.

Este consentimiento informado cumple con los lineamientos establecidos en el Reglamento de la Ley General de Salud en Materia de Investigación para la Salud, la Declaración de Helsinki y las Buenas Prácticas Clínicas emitidas por la Comisión Nacional de Bioética. Dicho consentimiento puede contener palabras que Ud. no comprenda; si es así, por favor pregunte a los psicólogos responsables para que le expliquen la palabra o información que no entienda claramente.

Ud. ha sido invitado(a) a participar en una intervención psicológica grupal. Antes de aceptar participar, lea por favor este formato de consentimiento cuidadosamente. Haga todas las preguntas que desee para asegurarse de que entiende los procedimientos del estudio, incluyendo sus riesgos y beneficios.

Participantes: La participación es completamente voluntaria. Puede permanecer o abandonar la intervención en cualquier momento sin que ello signifique una afectación de la atención médica que le proporciona la institución de salud. Para este proyecto, se tendrá en cuenta que sea beneficiario(a) del Hospital Regional "Gral. Ignacio Zaragoza”, ISSSTE, con diagnóstico de diabetes mellitus tipo 2 .

Tiempo requerido: Quincenalmente se llevará a cabo una sesión psicoterapéutica con una duración de 120 minutos.

Beneficios: Un beneficio que usted puede obtener al participar en el protocolo de intervención es que se le refiera, si usted así lo desea, a recibir una atención especializada para continuar con su tratamiento psicológico.

Con su participación, la investigación contribuirá a un mejor abordaje del tratamiento para así optimizar su proceso de atención a pacientes con diabetes mellitus tipo 2. Esta investigación no busca obtener beneficio económico alguno para el investigador ni para los colaboradores del estudio. Si usted decide participar, tendrá derecho a exigir toda la información al respecto, los resultados obtenidos y los análisis que se derivaron de la información registrada durante todo el proceso.

Privacidad y confidencialidad: El proceso es estrictamente confidencial. Su nombre no será utilizado en ningún informe cuando los resultados de la investigación sean publicados.

Derecho a retirarse del estudio de investigación: Puede retirarse del estudio en cualquier momento. Sin embargo, los datos obtenidos hasta ese momento seguirán formando parte del estudio, a menos que usted solicite expresamente que su identificación y su información sea borrada de nuestra base de datos.

Las preguntas o dudas sobre los derechos como participante en este proyecto pueden dirigirse a la

Lic. Patricia Zamudio, Hospital Regional "Gral. Ignacio Zaragoza”, ISSSTE.

\section{Investigadores principales:}

Psic. Sandra Nayeli Vergara Aguirre, Facultad de Estudios Superiores Zaragoza, UNAM.

Psic. Omar Arturo Chavarría Santiago, Facultad de Psicología, UNAM. 